Journal of Advanced Research in Fluid Mechanics and Thermal Sciences

Journal homepage: www.akademiabaru.com/arfmts.html ISSN: 2289-7879

\title{
The Effects of Weightage Values with Two Objective Functions in iPSO for Electro-Hydraulic Actuator System
}

\author{
Chai Mau Shern ${ }^{1}$, Rozaimi Ghazali ${ }^{1,}{ }^{*}$, Chong Shin Horng ${ }^{1}$, Chong Chee Soon ${ }^{1}$, Muhamad Fadli \\ Ghani $^{1,2}$, Yahaya Md Sam ${ }^{3}$, Zulfatman Has ${ }^{4}$ \\ 1 Centre for Robotics and Industrial Automation, Faculty of Electrical Engineering, Universiti Teknikal Malaysia Melaka, Hang Tuah Jaya, 76100 \\ Durian Tunggal, Melaka, Malaysia \\ 2 Malaysian Institute of Marine Engineering Technology (MIMET), Universiti Kuala Lumpur, Lumut, Perak, Malaysia \\ 3 Control \& Mechatronics Engineering, Faculty of Electrical Engineering, Universiti Teknologi Malaysia, 81310 Skudai, Johor Bahru, Malaysia \\ 4 Electrical Engineering Department, University of Muhammadiyah Malang, 65144 Malang, Indonesia
}

\section{ARTICLE INFO}

Article history:

Received 4 September 2020

Received in revised form 20 February 2021

Accepted 21 February 2021

Available online 26 March 2021

\section{Keywords:}

Electro-hydraulic Actuator System; Improved Particle Swarm Optimization; Linear Weight Summation

\section{ABSTRACT}

\begin{abstract}
In this paper, the Proportional-Integral-Derivative (PID) controller with improved Particle Swarm Optimization (iPSO) algorithm is proposed for the positioning control of nonlinear Electro-Hydraulic Actuator (EHA) system. PID controller is chosen to control the EHA system due to its popularity in industrial applications. The PID controller parameters will be tuned by using the iPSO algorithm to get the lowest overshoot percentage and steady-state error. The conventional PSO algorithm has only one objective function to get the optimum parameters. However, this is not enough to increase the control performance of the EHA system. Therefore, an improved Particle Swarm Optimization (iPSO) that includes the mean error and overshoot percentage as the two objective functions is proposed in this paper. The most popular method in PSO that included two objective functions is Linear Weight Summation (LWS). In this method, the two objective functions are combined with certain weightage into one equation to give the best control performance. This paper focuses on determining the suitable weightage between these two objective functions so that the EHA system can produce the best control performance with less overshoot and less error. Overshoot percentage and steady-state error are used to indicate the best control performance. The results showed that EHA system can perform better by using suitable weightage between the mean error and overshoot percentage.
\end{abstract}

\section{Introduction}

Electro-Hydraulic Actuator (EHA) system is widely applied in industrial applications due to its tremendous advantages such as small size to power ratio, high precision, and fast response [1-3]. However, the EHA system is known as a nonlinear system due to its nonlinear dynamic characteristics such as leakage, flow-pressure relationship, temperature, the friction of the valve, dead zone [4]. For

\footnotetext{
* Corresponding author.

E-mail address: rozaimi.ghazali@utem.edu.my
}

https://doi.org/10.37934/arfmts.81.2.98109 
that reason, the EHA system often requires a high control performance with high precision and heavy load so that it can produce a promising output in different industry applications [5].

Several types of control approaches had been proposed by researchers to control the nonlinear EHA system, such as linear quadratic regulator [6-8], PID controller [9-11], model predictive controller [12-14], backstepping controller [15-17], sliding mode controller [18-21], and other hybrid controllers [22-24]. However, the PID controller is still the main choice for industrial applications due to its fast design process, simple structure, and robust control performance [25]. In [9], a PID controller had been proposed to control an electro-hydraulic actuator and the control performance has been compared with a pole placement controller. Another PID controller with a second-order differential term had been proposed in [10] to control the electro-hydraulic actuator. A comparison study between a fuzzy controller and the PID controller was done in [11] and the results showed that the PID controller had faster tracking ability than the fuzzy controller.

On the other hand, it was found out that the nonlinearities existed in EHA system have made the control performance unsatisfactory with just a classical PID controller [26]. Therefore, several efforts have been tried to improve the PID controller performance, such as combine PID controller with other controllers, obtain the suitable PID controller parameters using optimization techniques, and improve the PID controller algorithm. The effective method is to obtain the most suitable PID controller parameters to give the best control performance. Early years, the trial-and-error method was used to tune the PID controller but the control performance was not guaranteed. Another famous PID tuning method called the Ziegler-Nichols method was proposed. Unfortunately, this method was shown to be aggressive and lead to large overshoot and oscillatory response [25].

Many researchers began to use the meta-heuristic optimization to obtain the PID controller parameters. One of the popular methods is Particle Swarm Optimization (PSO). PSO is much simpler and convenient as compared to other optimization techniques such as genetic algorithm [27]. The conventional PSO algorithm contains only one objective function which is not enough to increase the control performance of the EHA system. Therefore, an improved Particle Swarm Optimization (iPSO) that consist of two objective functions will be proposed to obtain the most suitable PID controller to give the best control performance. The two selected objective functions are mean error and overshoot percentage. This paper will mainly focus on the investigation of different weightage between these two objective functions and the PID controller parameters obtain through the best combination of the weightage will be selected.

The rest of the paper will be organized as follow: Section II will introduce the EHA system modelling, followed by the proposed control strategy in Section III. Section IV will present the implementation of the simulation study using MATLAB/Simulink and the results. Finally, a conclusion is presented and future recommendation is outlined in Section V.

\section{EHA System Modelling}

An established EHA system designed by Mete Kalyoncu and Mustafa Haydim is used in this paper [28]. Figure 1 shows the physical model of the EHA system utilized in this research. The motion of the hydraulic cylinder is controlled by the hydraulic oil flow from the cylinder chambers using a servo valve. The load is attached with a spring and a damper that generates a counterforce against the hydraulic cylinder. 


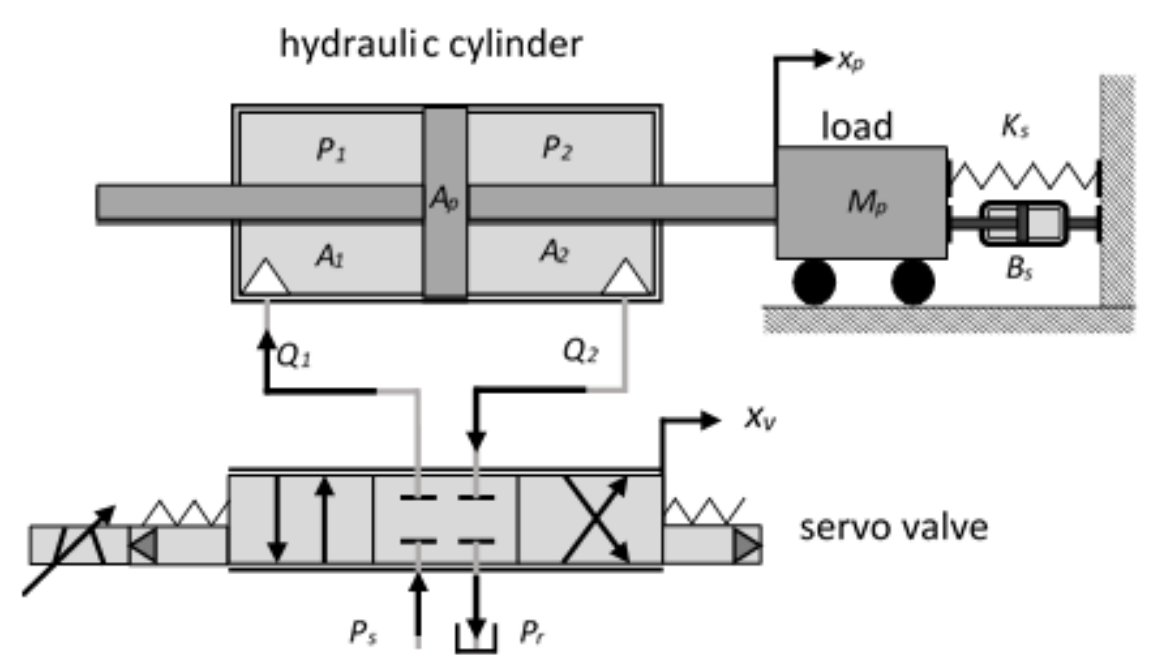

Fig. 1. The physical modelling of the EHA system

The electrical signal that drives the electric current to be used in the servo valve coil as represented in Eq. (1).

$V=\frac{d l}{d t} L_{c}+R_{c} I$

The dynamic of the servo valve in Eq. (2) is considered as a second-order system relates the current drive from the torque motor and the spool valve position.

$\frac{d^{2} x_{v}}{d t^{2}}+2 \xi \omega_{n} \frac{d x_{v}}{d t}+\omega_{n}^{2}=I \omega_{n}^{2}$

The flow relations for each chamber are presented in Eq. (4) and Eq. (5). The internal leakages effect in the servo valve is neglected.

$Q_{1}=\left\{\begin{array}{l}K_{1} x_{v} \sqrt{P_{s}-P_{1}} x_{v} \geq 0 \\ K_{1} x_{v} \sqrt{P_{1}-P_{r}} x_{v}<0\end{array}\right\}$

$Q_{2}=\left\{\begin{array}{l}-K_{2} x_{v} \sqrt{P_{2}-P_{r}} x_{v} \geq 0 \\ -K_{2} x_{v} \sqrt{P_{s}-P_{2}} x_{v}<0\end{array}\right\}$

where the coefficient gain $K_{1}=K_{2}=K$ for a symmetrical servo valve.

The volumes for each of the chamber in the hydraulic cylinder are as follows

$V_{1}=V_{\text {line }}+A_{p}\left(x_{s}+x_{p}\right)$

$V_{2}=V_{\text {line }}+A_{p}\left(x_{s}-x_{p}\right)$

where $V_{\text {line }}$ is the volume of the pipeline. 
The pressures for each chamber are presented in Eq. (7) and Eq. (8) by defining the relation between the flow rate, bulk modulus, external leakage and the volume rate.

$$
\begin{aligned}
& P_{1}=\frac{\beta}{V_{\text {line }}+A_{p}\left(x_{s}+x_{p}\right)} \int\left(Q_{1}-q_{12}-q_{1}-\frac{d V_{1}}{d t}\right) d t \\
& P_{2}=\frac{\beta}{V_{\text {line }}+A_{p}\left(x_{s}-x_{p}\right)} \int\left(\frac{d V_{2}}{d t}-q_{21}-q_{2}-Q_{2}\right) d t
\end{aligned}
$$

The total force produced to move the hydraulic cylinder can be expressed in Eq. (9) from the overall dynamic equation.

$$
F_{p}=A_{p}\left(P_{1}-P_{2}\right)=M_{p} \frac{d^{2} x_{p}}{d t^{2}}+B_{s} \frac{d x_{p}}{d t}+K_{s} x_{p}
$$

The parameters of the EHA system used in this paper have been tabulated in Table 1.

Table 1

The EHA system parameters

\begin{tabular}{lll}
\hline Symbol & Description & Value \\
\hline$I_{\text {sat }}$ & Torque motor saturation current & $0.02 \mathrm{~A}$ \\
$L_{c}$ & Servo-valve coil inductance & $0.59 \mathrm{H}$ \\
$R_{c}$ & Servo-valve coil resistance & $100 \Omega$ \\
$b$ & Hydraulic fluid bulk modulus & $1.4 \times 10^{9} \mathrm{~N} / \mathrm{m}^{2}$ \\
$\omega_{n}$ & Servo-valve natural frequency & $534 \mathrm{rad} / \mathrm{s}$ \\
$\xi$ & Servo-valve damping ratio & 0.48 \\
$P_{r}$ & Return pressure & $0 \mathrm{~Pa}$ \\
$P_{s}$ & Pump pressure & $2.1 \times 10^{7} \mathrm{~Pa}$ \\
$K$ & Servo-valve gain & $2.38 \times 10^{-5} \mathrm{~m}^{5 / 2} / \mathrm{kg}^{1 / 2}$ \\
$M_{p}$ & Total mass & $9 \mathrm{~kg}$ \\
$X_{s}$ & Total actuator displacement & $0.1 \mathrm{~m}$ \\
$B_{s}$ & Damping coefficient & $2000 \mathrm{Ns} / \mathrm{m}$ \\
$A_{p}$ & Piston area & $645 \times 10^{-6} \mathrm{~m}^{2}$ \\
$K_{s}$ & Spring stiffness & $10 \mathrm{Nm}$ \\
\hline
\end{tabular}

\section{Proposed Control Strategy}

\subsection{Proportional-Integral-Derivative (PID) Controller}

PID controller is the most popular controller in industry applications. The overall PID control function can be expressed mathematically as in Eq. (10).

$u(t)=K_{P} e(t)+K_{l} \int_{0}^{t} e(t) d t+K_{D} \frac{d e(t)}{d t}$

Figure 2 illustrates the PID controller structure used in this paper. The top path is called the proportional path, the output of the proportional path is the multiplication of the error $(e)$ and the proportional gain, $K_{p}$. The second path is the integral path. The output of this path is the multiplication of the integral of the error $(e)$ and the integral gain, $K_{l}$. Note that the integral of the 
error is the area under the curve of the graph of error $(e)$ versus time. Finally, the third path is the derivative path. The error $(e)$ is first differentiated to get the rate of change of the error and then multiplied it with derivative gain, $K_{D}$. All the output of these three paths is then added together using a summing block to become a total PID controller action and produce a control signal $(u)$ to a plant or system.

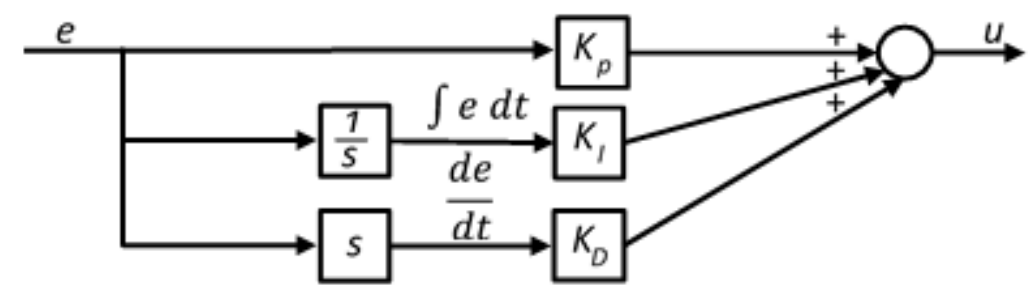

Fig. 2. The PID controller structure

\subsection{Particle Swarm Optimization (PSO)}

Conventional PSO is an optimization method introduced by Kennedy and Eberhart in 1995 [29]. This algorithm was motivated by the social behaviour of organisms such as fish schooling and bird flocking to search for the food. In PSO, the particles population is called the swarm and its potential solutions are called particles. The particles flying around in a multidimensional problem space [30].

First, the initial position of a particle is initialized using Eq. (11). Each potential solution of the optimization problem is represented by a single particle.

$$
x^{i}=x_{\min }+\left(x_{\max }-x_{\min }\right) \times \text { rand }
$$

where $x^{i}$ is the position of the particle at $i$ order, $x_{\min }$ and $x_{\max }$ are the minimum and maximum values in the search space, rand is randomly generated number by using MATLAB between 0 and 1 . Then the best position achieved by each particle based on its own experience will set as local best, $P_{B E S T}$ and the best position achieved by a group of particles in the entire swarm will be set as global best, GBEST.

The position and velocity of each particle will change with time (iteration). The new position, $x^{i+1}$ and new velocity, $v^{i+1}$ of each particle in every iteration are updated using Eq. (12) and Eq. (13).

$$
\begin{aligned}
& x^{i+1}=x^{i}+v^{i+1} \\
& v^{i+1}=\omega v^{i}+c_{1} r_{1}\left(P_{\text {BEST }}^{i}-x^{i}\right)+c_{2} r_{2}\left(G_{\text {BEST }}-x^{i}\right)
\end{aligned}
$$

where $w$ is the linearly decreasing inertia weight, $v^{i}$ is the current velocity of the particle, $r_{1}, r_{2}$ are the random numbers that uniformly distributed in the interval 0 to $1, c_{1}$ and $c_{2}$ are acceleration constants.

To improve the accuracy and efficiency, a linearly decreasing inertia weight from $w_{\max }$ to $w_{\min }$ as shown in Eq. (14) is applied.

$$
w=w_{\max }-\left(\frac{\left(w_{\max }-w_{\min }\right)}{i}\right) \times \max i t e r
$$

where $w_{\max }$ and $w_{\min }$ are the boundaries on inertia weight, maxiter is the maximum iteration number. 


\subsection{Improved Particle Swarm Optimization (iPSO)}

The conventional PSO was devised only to solve for single-objective problems [31]. An improved Particle Swarm Optimization (iPSO) that consists of two objective functions was proposed in this paper to optimize more than one objective function throughout the whole optimization. Two selected objective functions which are mean error and overshoot percentage utilized in this paper are as shown in Eq. (15) and Eq. (16).

$$
\text { mean_error }=\text { mean(error) }
$$

overshoot_percentage $=\left(\frac{\max (\text { output })-\text { input }}{\text { input }}\right) \times 100 \%$

Among the available PSO algorithm with more than one objective function [25, 27, 32-37], linear weight summation (LWS) approach or another named weight aggregation (WA) strategy is the simplest and most popular method. This method converts a multi-objective problem into a single fitness equation as in Eq. (17) using specific or selected weightage.

$$
\text { Fitness }=\alpha(\text { mean_error })+\beta \text { (overshoot_percentage })
$$

where $\alpha$ and $\beta$ are two weightage for the trade-offs between the mean error and the overshoot percentage, and $\alpha+\beta=1$. Higher weightage value means higher priority is placed on the respective objective function. Mean error and overshoot values in Eq. (17) are normalized values of the result obtained from Eq. (15) and Eq. (16).

To get the optimum PID controller parameters, different combinations of $\alpha$ and $\beta$ are tested and the output control performance of the EHA system is analyzed and the results are shown in the next section. Table 2 shows the different combinations of weightage values selected in this paper.

Table 2

Different combinations of weightage values

\begin{tabular}{lll} 
& Combinations & \multicolumn{2}{c}{ Weightage Values } \\
\cline { 2 - 3 } Case & $\alpha$ & $\beta$ \\
\hline 1 & 0.1 & 0.9 \\
2 & 0.2 & 0.8 \\
3 & 0.3 & 0.7 \\
4 & 0.4 & 0.6 \\
5 & 0.5 & 0.5 \\
6 & 0.6 & 0.4 \\
7 & 0.7 & 0.3 \\
8 & 0.8 & 0.2 \\
9 & 0.9 & 0.1 \\
\hline
\end{tabular}

Case 1 to case 4 indicates that more weight on $\beta$, which means that more priority is put on overshoot percentage. Case 5 shows equally weight factor for both $\alpha$ and $\beta$, and this means that mean error and overshoot percentage are equally important and is taken into consideration equally during our simulation. Case 6 to case 9 show more weight on $\alpha$, and this indicates that more priority is put on a mean error in our optimization throughout the whole simulation. 


\section{Implementation, Results and Discussion}

All the simulations are conducted using Intel (R) Core (TM) i7-4790 Processor, 16.0 GB RAM, 3.60 $\mathrm{GHz}$, Microsoft Windows 7 and MATLAB version 2016b. The EHA system model with nonlinear equations in [28] is designed via MATLAB/Simulink as shown in Figure 3.

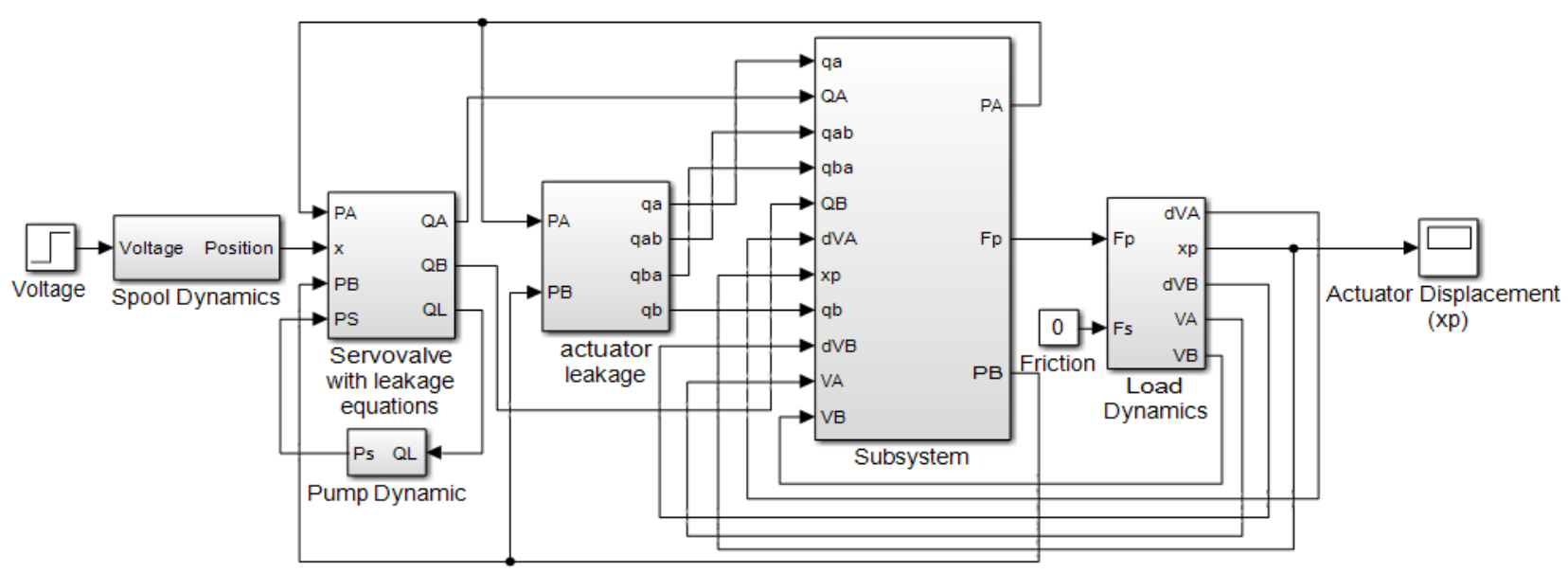

Fig. 3. The structure of the EHA system model

A complete control structure that includes the EHA system model and a PID controller is illustrated in Figure 4. An input voltage corresponding to position input (reference step input) is transmitted to the PID controller optimized using the proposed iPSO algorithm. The input current is generated in proportion to the error between voltage output and the voltage input to fed into the EHA system. Time-domain specifications such as mean error, overshoot percentage, and steady-state error will be analyzed.

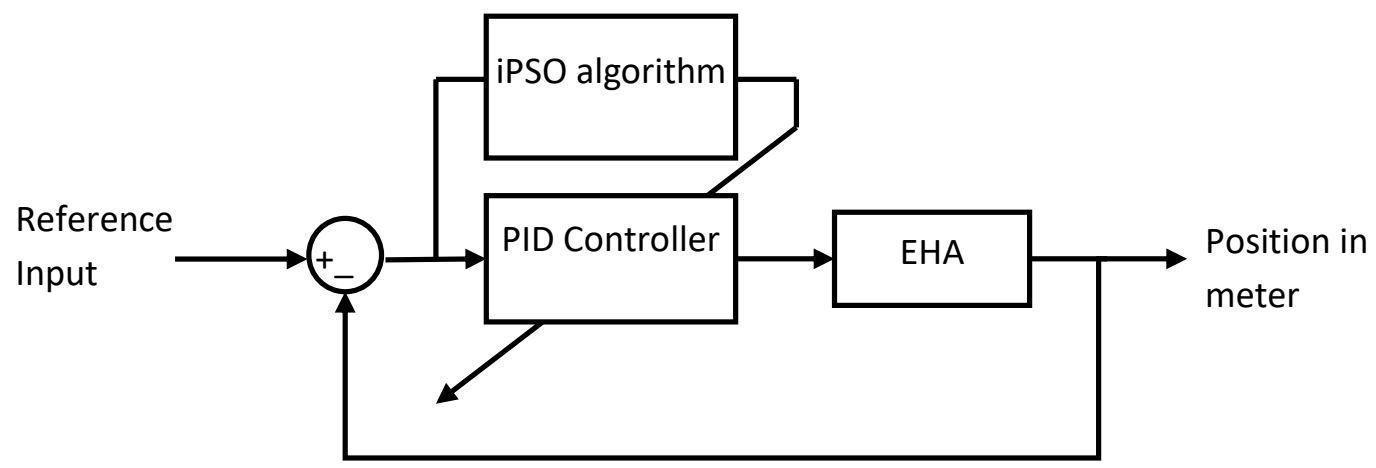

Fig. 4. The control structure of the EHA system model and a PID controller with iPSO

The parameters setting of iPSO is shown in Table 3. Each case in Table 2 runs for 5 times, and the average value is selected for the result analysis.

The parameters of PID controller $K_{P}, K_{1}$, and $K_{D}$ obtain through the iPSO algorithm for each case is shown in Table 4. The parameters of the PID controller are the average values obtained from 5 runs for each case.

After obtaining the controller parameters using the proposed iPSO, the controller parameters recorded in Table 4 are then used in the PID controller and the EHA system (shown in Figure 5) is simulated again. 


\section{Table 3}

Optimization parameters

\begin{tabular}{lll}
\hline Symbol & Description & Value \\
\hline$c_{1}$ & Cognitive component & 2.0 \\
$c_{2}$ & Social component & 2.0 \\
$N$ & Number of populations & 30 \\
$d i m$ & Dimension & 3 \\
maxiter & Number of iterations in each optimization & 50 \\
$x_{\min }$ & Minimum values in search space boundary & 0 \\
$x_{\max }$ & Maximum value in search space boundary & 30 \\
$w_{\max }$ & Initial weight value & 0.9 \\
$w_{\min }$ & Final weight value & 0.4 \\
\hline
\end{tabular}

Table 4

Controller parameters for each case

\begin{tabular}{llll}
\hline \multirow{2}{*}{ Case } & \multicolumn{3}{l}{ Controller Parameters } \\
& $K_{P}$ & $K_{l}$ & $K_{D}$ \\
\hline 1 & 29.1899 & 0.0383 & 0.0340 \\
2 & 29.8985 & 0.0311 & 0.1789 \\
3 & 29.7331 & 0.0250 & 0.4219 \\
4 & 29.9736 & 0.8924 & 0.1623 \\
5 & 29.9542 & 0.0091 & 0.2654 \\
6 & 29.6628 & 0.0082 & 0.0090 \\
7 & 29.6211 & 0.1035 & 0.0338 \\
8 & 29.6781 & 0.0017 & 0.4333 \\
9 & 29.8833 & 0.6325 & 0.4028 \\
\hline
\end{tabular}

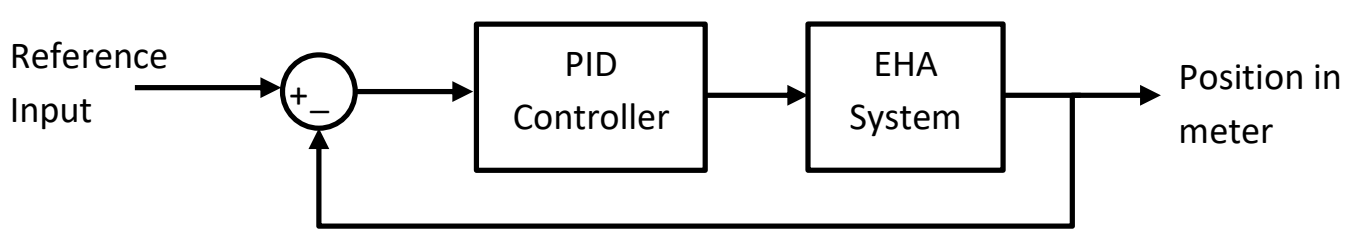

Fig. 5. The block diagram of the EHA system with a PID controller

The output performances of the EHA system are illustrated in Figure 6 and Figure 7. Figure 6 illustrates the steady-state error while Figure 7 shows the overshoot of the EHA system. The simulation time is 10 seconds. A $0.03 \mathrm{~m}$ of displacement act as the step reference input signal is fed into the EHA system at step time equal to 3 seconds to evaluate the position tracking performance. The sampling time used in the simulation in this paper is 0.001 second.

The time-domain specifications such as mean error, overshoot percentage, and steady-state error are analyzed and recorded. Table 5 shows the output performance for each case of the combination of weight values using the PID controller parameters in Table 4.

From Table 5, mean errors for all the cases do not show much difference. The mean error for case 1 to case 9 are in between $0.635 \times 10^{-3} \mathrm{~m}$ and $0.908 \times 10^{-3} \mathrm{~m}$, which are only below 1 millimetre. For the overshoot percentage, case 2 showed the highest overshoot percentage, which is $1.7057 \%$ while case 7 showed the lowest overshoot percentage, which is $0.0362 \%$. Apart from that, case 7 showed a result of $1.081 \times 10^{-5} \mathrm{~m}$ in steady-state error, which is the lowest among all the cases shown in Table 5. 


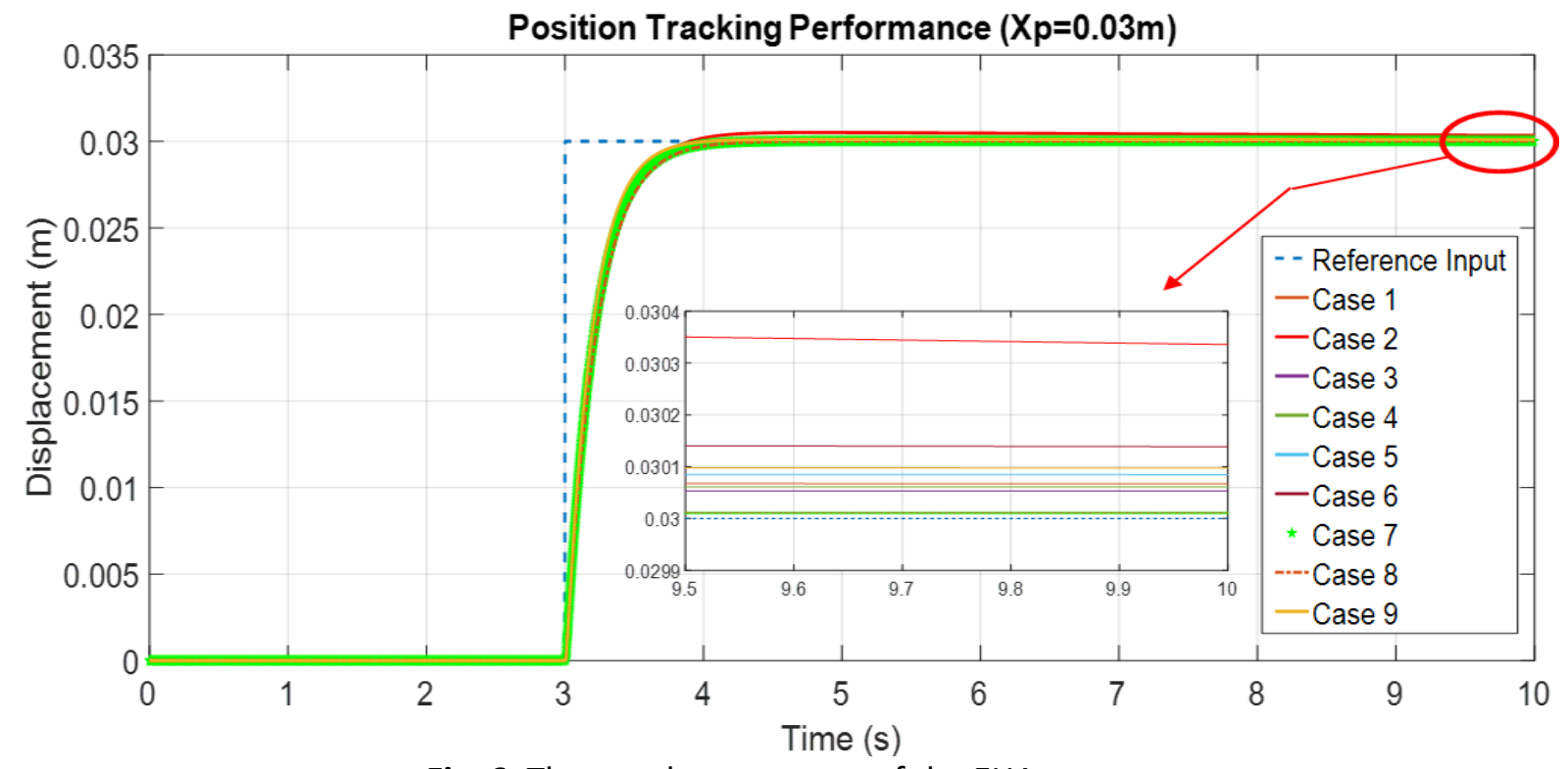

Fig. 6. The steady-state error of the EHA system

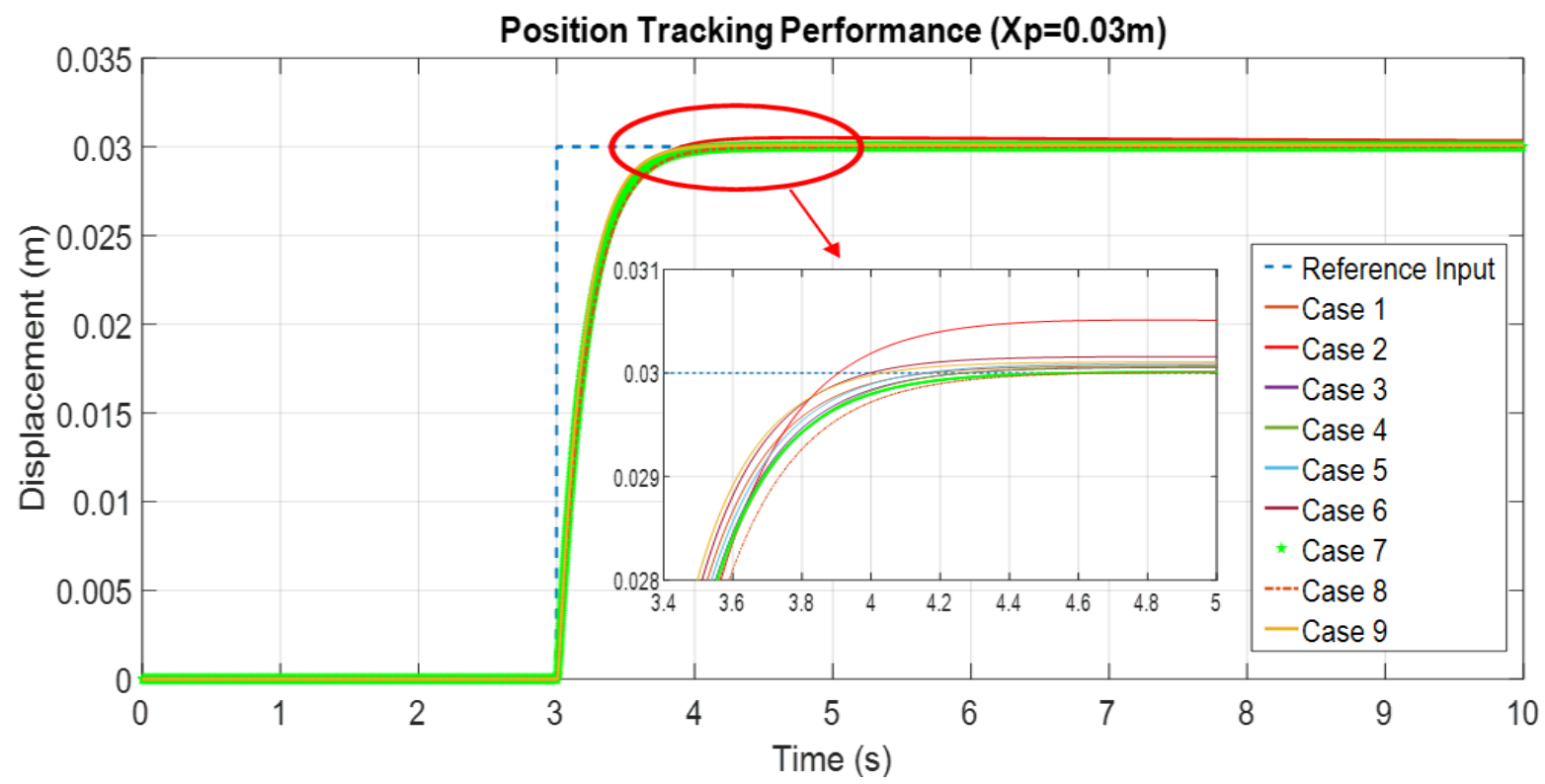

Fig. 7. The overshoot of the EHA system

Table 5

The output performance of the EHA system

\begin{tabular}{llll}
\hline \multirow{2}{*}{ Case } & \multicolumn{2}{l}{ Output Performance } & \\
\cline { 2 - 4 } & Mean Error $(\mathrm{m})$ & Overshoot Percentage $(\%)$ & Steady-state Error $(\mathrm{m})$ \\
\hline 1 & $0.645 \times 10^{-3}$ & 0.2360 & $6.712 \times 10^{-5}$ \\
2 & $0.908 \times 10^{-3}$ & 1.7057 & $33.573 \times 10^{-5}$ \\
3 & $0.659 \times 10^{-3}$ & 0.1837 & $5.307 \times 10^{-5}$ \\
4 & $0.674 \times 10^{-3}$ & 0.2121 & $6.096 \times 10^{-5}$ \\
5 & $0.666 \times 10^{-3}$ & 0.3017 & $8.460 \times 10^{-5}$ \\
6 & $0.678 \times 10^{-3}$ & 0.5275 & $13.853 \times 10^{-5}$ \\
7 & $0.639 \times 10^{-3}$ & 0.0362 & $1.081 \times 10^{-5}$ \\
8 & $0.678 \times 10^{-3}$ & 0.0383 & $1.143 \times 10^{-5}$ \\
9 & $0.635 \times 10^{-3}$ & 0.3540 & $9.709 \times 10^{-5}$ \\
\hline
\end{tabular}


Among all the case with different combinations of weight factors in iPSO, case 7 with the weight factors of 0.7 on a mean error and 0.3 on overshoot is found to be the best combination. Simulation results showed that under this combination, the EHA system obtained the least overshoot percentage and steady-state error. Therefore, it can be concluded that the mean error is more important or having a higher priority in selecting the optimal PID controller parameters.

\section{Conclusions}

This paper presents a control strategy that combines the PID controller and an improve PSO for positioning control in the EHA system. Also, the nonlinear dynamic equations of the EHA system has been derived and explained. In this paper, the proposed iPSO based on linear weight summation has been used to find suitable PID controller parameters. Time-domain analysis has been examined. Simulation results have shown that the EHA system performed better by using the best combination of weightage (mean error and overshoot is 0.7:0.3).

In the future, different types of controllers will be considered. More advanced optimization techniques such as cuckoo search, bat algorithm, firefly algorithm, and grey wolf optimizer will be used to optimize the controller parameters.

\section{Acknowledgement}

The support of Universiti Teknikal Malaysia Melaka (UTeM), Centre for Robotics and Industrial Automation (CeRIA), and Ministry of Education (MOE) are greatly acknowledged. The research was funded by the Fundamental Research Grant Scheme (FRGS) Grant No. (FRGS/1/2017/TK04/FKECeRIA/F00333).

\section{References}

[1] Ghazali, Rozaimi, Chong Chee Soon, Chong Shin Horng, Chai Mau Shern, Yahaya Md Sam, and Ahmad Anas Yusof. "Controllers Capabilities with Computational Tuning Algorithm in Nonlinear Electro-Hydraulic Actuator System." Journal of Advanced Research in Fluid Mechanics and Thermal Sciences 52, no. 2 (2018): 148-160.

[2] Rehman, Waheed Ur, Shaoping Wang, Xingjian Wang, Lei Fan, and Kamran Ali Shah. "Motion synchronization in a dual redundant HA/EHA system by using a hybrid integrated intelligent control design." Chinese Journal of Aeronautics 29, no. 3 (2016): 789-798. https://doi.org/10.1016/i.cja.2015.12.018

[3] Dong, Meng, Xiting Luan, Baoyuan Wu, and Junlong Liang. "The Fuzzy Control of Electro-hydraulic Servo System Based on DE Algorithm." In Proceedings of 2018 Chinese Intelligent Systems Conference, pp. 747-757. Springer, Singapore, 2019. https://doi.org/10.1007/978-981-13-2291-4 73

[4] Guo, Qinyang, Guanglin Shi, and Dongmei Wang. "Adaptive Composite Fuzzy Dynamic Surface Control for ElectroHydraulic-System, with Variable-Supply-Pressure." Asian Journal of Control 22, no. 1 (2020): 521-535. https://doi.org/10.1002/asjc.1907

[5] Hoang, Quoc-Dong, Soon-Geul Lee, and Batsaikhan Dugarjav. "Super-twisting observer-based integral sliding mode control for tracking the rapid acceleration of a piston in a hybrid electro-hydraulic and pneumatic system." Asian Journal of Control 21, no. 1 (2019): 483-498. https://doi.org/10.1002/asjc.1971

[6] Pourebrahim, Majid, Ali Selk Ghafari, and Mahshid Pourebrahim. "Designing a LQR controller for an electrohydraulic-actuated-clutch model." In 2016 2nd International Conference on Control Science and Systems Engineering (ICCSSE), pp. 82-87. IEEE, 2016. https://doi.org/10.1109/CCSSE.2016.7784358

[7] Ahmed, Mazid Ishtique, and A. K. M. Azad. "Mathematical modeling and DLQR based controller design for a nonminimum phase Electro Hydraulic Servo system (EHS)." In 2016 IEEE Region 10 Conference (TENCON), pp. 18391844. IEEE, 2016. https://doi.org/10.1109/TENCON.2016.7848339

[8] Shaaban, Sayed, Mohamed Rabie, Magdy Roman, and Mohamed Aly. "Observer-Based Optimal Position Control for Electrohydraulic Steer-by-Wire System Using Gray-Box System Identified Model." ASME, 2017. https://doi.org/10.1115/1.4037164

[9] Adnan, Ramli, Mazidah Tajjudin, Norlela Ishak, Hashimah Ismail, and Mohd Hezri Fazalul Rahiman. "Self-tuning fuzzy PID controller for electro-hydraulic cylinder." In 2011 IEEE 7th International Colloquium on Signal Processing and its Applications, pp. 395-398. IEEE, 2011. https://doi.org/10.1109/CSPA.2011.5759909 
[10] Qiankun, Ma, Wang Xuyong, Yuan Fan, Tao Jianfeng, and Liu Peng. "Research on feed-forward PIDD 2 control for hydraulic continuous rotation motor electro-hydraulic servo system with long pipeline." In 2016 UKACC 11th International Conference on Control (CONTROL), pp. 1-6. IEEE, 2016.

[11] Wang, Xiangdong, Dongxu Wang, Shujing Li, and Xue Li. "Design of position balance controller for the sprayer boom." In 2018 3rd International Conference on Mechanical, Control and Computer Engineering (ICMCCE), pp. 304307. IEEE, 2018. https://doi.org/10.1109/ICMCCE.2018.00069

[12] Essa, Mohamed El-Sayed M., Magdy AS Aboelela, M. A. Moustafa Hassan, and S. M. Abdrabbo. "Model predictive force control of hardware implementation for electro-hydraulic servo system." Transactions of the Institute of Measurement and Control 41, no. 5 (2019): 1435-1446. https://doi.org/10.1177/0142331218784118

[13] Liang, Xuan Wong, and Zool Hilmi Ismail. "System Identification and Model Predictive Control using CVXGEN for Electro-Hydraulic Actuator." International Journal of Integrated Engineering 11, no. 4 (2019). https://doi.org/10.30880/ijie.2019.11.04.018

[14] Liu, Shengfei, Qinglin Sun, Zengqiang Chen, and Fangyou Dong. "Characteristic model-based generalized predictive control for electro-hydraulic system with dead-zones and parameter uncertainty." IFAC-PapersOnLine 51, no. 20 (2018): 287-294. https://doi.org/10.1016/i.ifacol.2018.11.027

[15] Yang, Xuebo, Xiaolong Zheng, and Yuhong Chen. "Position tracking control law for an electro-hydraulic servo system based on backstepping and extended differentiator." IEEE/ASME Transactions on Mechatronics 23, no. 1 (2017): 132-140. https://doi.org/10.1109/TMECH.2017.2746142

[16] Guo, Qing, Jing-min Yin, Tian Yu, and Dan Jiang. "Coupled-disturbance-observer-based position tracking control for a cascade $\quad$ electro-hydraulic $\quad$ system." ISA $\quad$ transactions $68 \quad$ (2017): $367-380$. https://doi.org/10.1016/i.isatra.2017.02.014

[17] Guo, Qing, Ping Sun, Jing-min Yin, Tian Yu, and Dan Jiang. "Parametric adaptive estimation and backstepping control of electro-hydraulic actuator with decayed memory filter." ISA transactions 62 (2016): $202-214$. https://doi.org/10.1016/i.isatra.2016.02.009

[18] Lee, Dongyoung, Buchun Song, Sang Yong Park, and Yoon Su Baek. "Development and Control of an ElectroHydraulic Actuator System for an Exoskeleton Robot." Applied Sciences 9, no. 20 (2019): 4295. https://doi.org/10.3390/app9204295

[19] Cheng, Lei, Zhen-Cai Zhu, Gang Shen, Shujing Wang, Xiang Li, and Yu Tang. "Real-time force tracking control of an electro-hydraulic system using a novel robust adaptive sliding mode controller." IEEE Access 8 (2019): 1331513328. https://doi.org/10.1109/ACCESS.2019.2895595

[20] Kang, Shuo, Hao Yan, Lijing Dong, and Changchun Li. "Finite-time adaptive sliding mode force control for electrohydraulic load simulator based on improved GMS friction model." Mechanical Systems and Signal Processing 102 (2018): 117-138. https://doi.org/10.1016/i.ymssp.2017.09.009

[21] Jiangtao, Feng, Gao Qinhe, Guan Wenliang, and Chen Zhixiang. "Adaptive fuzzy sliding mode control for large erection mechanism." In 2017 36th Chinese Control Conference (CCC), pp. 3599-3604. IEEE, 2017.

[22] Tran, Duc Thien, Keunhui Jeong, Giho Jun, Jinho Suh, Maolin Jin, and Kyoung Kwan Ahn. "Adaptive gain backstepping sliding mode control for electrohydraulic servo system with uncertainties." In 2017 14th International Conference on Ubiquitous Robots and Ambient Intelligence (URAI), pp. 534-539. IEEE, 2017.

[23] Rozali, Sahazati Md, Nor Syaza Farhana, Muhammad Nizam Kamarudin, Amar Faiz Zainal Abidin, Mohd Fua'ad Rahmat, Abdul Rashid Husain, and Chong Chee Soon. "Robust control design of nonlinear system via BacksteppingPSO with sliding mode techniques." In Asian Simulation Conference, pp. 27-37. Springer, Singapore, 2017. https://doi.org/10.1007/978-981-10-6463-0 3

[24] Sun, Chungeng, Jinhui Fang, Jianhua Wei, and Bo Hu. "Nonlinear motion control of a hydraulic press based on an extended disturbance $\quad$ observer." leee $\quad$ Access $6 \quad$ (2018): https://doi.org/10.1109/ACCESS.2018.2813317

[25] Jaafar, Hazriq Izzuan, M. F. Sulaima, Z. Mohamed, and J. J. Jamian. "Optimal PID controller parameters for nonlinear gantry crane system via MOPSO technique." In 2013 IEEE Conference on Sustainable Utilization and Development in Engineering and Technology (CSUDET), pp. 86-91. IEEE, 2013. https://doi.org/10.1109/CSUDET.2013.6670992

[26] Zhou, Hua, Liming Lao, Yinglong Chen, and Huayong Yang. "Discrete-time sliding mode control with an input filter for an electro-hydraulic actuator." IET Control Theory \& Applications 11, no. 9 (2017): 1333-1340. https://doi.org/10.1049/iet-cta.2016.0951

[27] Sharaf, Adel M., and Adel AA El-Gammal. "A novel discrete multi-objective Particle Swarm Optimization (MOPSO) of optimal shunt power filter." In 2009 IEEE/PES Power Systems Conference and Exposition, pp. 1-7. IEEE, 2009. https://doi.org/10.1109/PSCE.2009.4839957

[28] Kalyoncu, Mete, and Mustafa Haydim. "Mathematical modelling and fuzzy logic based position control of an electrohydraulic servosystem with internal leakage." Mechatronics 19, no. 6 (2009): 847-858. https://doi.org/10.1016/i.mechatronics.2009.04.010 
[29] Kennedy, James, and Russell Eberhart. "Particle swarm optimization." In Proceedings of ICNN'95-international conference on neural networks, vol. 4, pp. 1942-1948. IEEE, 1995.

[30] Hazra, Jagabondhu, and Avinash K. Sinha. "Congestion management using multiobjective particle swarm optimization." IEEE Transactions on Power Systems 22, no. $4 \quad$ (2007): 1726-1734. https://doi.org/10.1109/TPWRS.2007.907532

[31] Chamaani, Somayyeh, S. Abdullah Mirtaheri, and Mohammad S. Abrishamian. "Improvement of time and frequency domain performance of antipodal Vivaldi antenna using multi-objective particle swarm optimization." IEEE Transactions on Antennas and Propagation 59, no. $5 \quad$ (2011): $1738-1742$. https://doi.org/10.1109/TAP.2011.2122290

[32] Kitamura, Shoichi, Kazuyuki Mori, Seiichi Shindo, Yoshio Izui, and Yoshihiko Ozaki. "Multiobjective energy management system using modified MOPSO." In 2005 IEEE International Conference on Systems, Man and Cybernetics, vol. 4, pp. 3497-3503. IEEE, 2005.

[33] Jaafar, Hazriq Izzuan, A. R. Abdullah, Z. Mohamed, J. J. Jamian, A. M. Kassim, M. S. M. Aras, M. N. M. Nasir, and M. F. Sulaima. "Analysis of Optimal Performance for Nonlinear Gantry Crane System using MOPSO with Linear Weight Summation Approach." In Proc. Malaysian Tech. Univ. Conf. Eng. Technol, vol. 5, pp. 1-6. 2013.

[34] Kang, Qi, ShuWei Feng, MengChu Zhou, Ahmed Chiheb Ammari, and Khaled Sedraoui. "Optimal load scheduling of plug-in hybrid electric vehicles via weight-aggregation multi-objective evolutionary algorithms." IEEE Transactions on Intelligent Transportation Systems 18, no. 9 (2017): 2557-2568.

[35] Elgammal, Adel Abdelaziz Abdelghany, and Mohammed Fathy El_Naggar. "MOPSO-based predictive control strategy for efficient operation of sensorless vector-controlled fuel cell electric vehicle induction motor drives." Turkish Journal of Electrical Engineering \& Computer Sciences 25, no. 4 (2017): $2968-2985$. https://doi.org/10.3906/elk-1608-115

[36] Mac, Thi Thoa, Cosmin Copot, Robin De Keyser, and Clara M. Ionescu. "The development of an autonomous navigation system with optimal control of an UAV in partly unknown indoor environment." Mechatronics 49 (2018): 187-196. https://doi.org/10.1016/i.mechatronics.2017.11.014

[37] Laware, A. R., D. B. Talange, and V. S. Bandal. "Evolutionary optimization of sliding mode controller for level control system." ISA transactions 83 (2018): 199-213. https://doi.org/10.1016/i.isatra.2018.08.011 